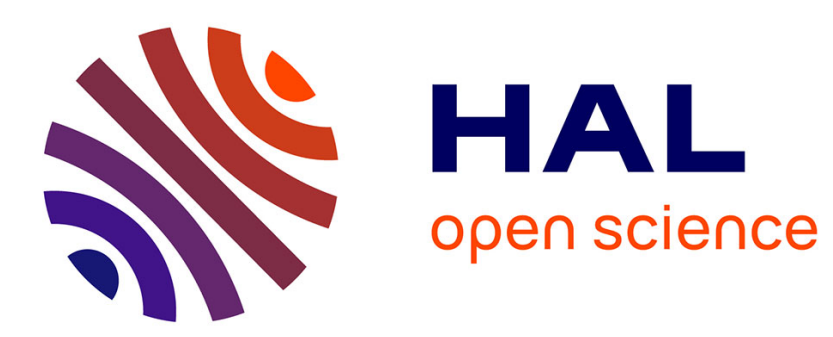

\title{
Inseln auf den Inseln. Grenzziehungen in Georg Forsters 'Reise um die Welt'
}

\author{
Anne Peiter
}

\section{To cite this version:}

Anne Peiter. Inseln auf den Inseln. Grenzziehungen in Georg Forsters 'Reise um die Welt'. Colloque "Inseln, Archipele, Atolle. Ordnungen des Insularen", Sep 2008, Mannheim, Germany. pp.169-185. hal-01157750

\section{HAL Id: hal-01157750 https://hal.univ-reunion.fr/hal-01157750}

Submitted on 23 Aug 2021

HAL is a multi-disciplinary open access archive for the deposit and dissemination of scientific research documents, whether they are published or not. The documents may come from teaching and research institutions in France or abroad, or from public or private research centers.
L'archive ouverte pluridisciplinaire HAL, est destinée au dépôt et à la diffusion de documents scientifiques de niveau recherche, publiés ou non, émanant des établissements d'enseignement et de recherche français ou étrangers, des laboratoires publics ou privés. 


\title{
Inseln auf den Inseln. Grenzziehungen in \\ Georg Forsters Reise um die We/t
}

\author{
ANNE D. PEITER
}

I.

In diesem Artikel soll es um die Bedeutung von Grenzziehungen für den Kontakt zwischen europäischen Reisenden und den Bewohnern unterschiedlicher Inseln im 18. Jahrhundert gehen. Diese Grenzziehung erfolgt, wie wir an unterschiedlichen Beispielen sehen werden, auf oft subtile Weise, gewinnt aber für die Etablierung von Machtstrukturen und die Herstellung einer gewissen Asymmetrie zwischen Einheimischen und Fremden große Bedeutung.

Georg Forster, geboren 1754. gestorben 1794. gelangte durch sein Buch Reise um die Welt bereits als junger Mann zu großer Berûhmtheit. Als Teilnehmer an Captain Cooks zweiter Weltumsegelung (1772-1775) hatte er die Gelegenheil, neben dem Kap Südafrikas und dem Südpol besonders die Inselwelt des Pazifik kennen zu lernen. Einer der Hauptaufträge Cooks war die Suche nach einem neuen, am Südpol vermuteten Kontinent. Der strapaziöse Versuch, trotz der Kälte immer weiter nach Süden vorzudringen, zeigte jedoch, dass die erwartete paradiesische Welt nicht zu finden war. In diesem Moment wurde ein Ausweichen nach Neuseeland, Tahiti und zu den Gesellschaftsinseln unumgänglich: Eine Pause war notwendig. Die Besatzung der beiden Schiffe konnte sich dort mit vitaminreicher Nahrung versorgen und Vorrāte für den zweiten Teil der Reise anlegen. der erneut der Suche nach dem erhofften, unbekannten Territorium galt. Dieser zweite Versuch war so erfolglos wie der erste. Ein weiteres Mal gewann daher die Inselwelt des Pazifik große Bedeutung. denn ohne Frūchte und Fleisch hätte die Besatzung die Rückkehr nach Europa nicht leisten können. Bei dieser zweiten Reise zu mehreren Inseln - darunter dieses Mal neben den Gesellschafts- und Freundschaftsinseln auch die Oster- und die 
Marquesasinseln - kam es zur Wiederbegegnung mit einigen Einheimischen.'

Dieser Kontext ist für die Analyse, die ich im Folgenden dem Kontakt zwischen den Europāern und den Bewohnern verschiedener Inseln widmen möchte, wichtig. Wichtig in zweifacher Hinsicht: Erstens ist zu unterstreichen, dass die Landung auf den Inseln nicht allein der Exploration fremder Welten galt, sondern ganz praktisch der Hoffnung, durch Tausch von Waren zu neuen Vorrăten zu gelangen. Und zweitens ist festzuhalten, dass es - bedingt auch durch Cooks erste Reise - auf manchen Inseln bereits regelrechte Traditionen gab, die die Kommunikation zwischen Europảern und Inselbewohnern in bestimmte Bahnen lenkten, dass aber der Modus des Miteinanders, des Austausches, des Kontaktes häufig erst noch festzulegen war. ${ }^{2}$ Mein Interesse soll im Folgenden besonders dem Austausch unter diesen Bedingungen gelten.

\section{II.}

Der erste Kontakt zwischen zwei Gruppen von Menschen, die unterschiedlichen Kulturen angehören und keine gemeinsame Sprache sprechen, ist zunächst einmal ein Wagnis. Die Europăer erwarten diese Begegnung; die Inselbewohner hingegen haben von der ersten Sichtung der fremden Schiffe bis zu ihrer Ankunft nur wenig Zeli, um sich auf die neue Situation vorzubereiten. Das, was sich bereits in den ersten Minuten abspielt, ist also durch eine Asymmetrie ge: kennzeichnet: Wăhrend die Europãer sich auf Begegnungen einstellen können, werden die Einheimischen plôtzlich aus ihrem Alltag herausgeríssen. Die Europâer kōnnen - nicht zuletzt militărische Vorsichtsmaßnahmen ergreifen. die Inselbewohner hingegen leben oft so zerstreut, dass sie ihre Kräfte bei entstehenden Konflikten erst bündeln mûssen.

Beim Blick auf die Beschreibungen, die Forster der ersten Kontaktaufnahme widmet, fâllt jedoch auf, dass es zu Beginn meist zu keiner Gewalt kommt. Das liegt vor allem daran, dass die Europãer das Ungleichgewicht, das zwischen thnen und den Fremden bezûg-

1 Vgl. zur Reiseroute und den Bedingungen der Reise: Ludwig Uhlig: Georg Forster. Lebensabenteuer eines gelehrten Weltburgers, Cottingen: Vandenhoeck \& Ruprecht 2004, besonders Kapitel III und IV.

2 Anja Schwarz unterstreicht in ihrem Aufsatz Linien im Sand, dass del Strand haufig als pkontextlose Urszenen " inszeniert wurde, dass aber in Wirklichkeit oft schon Kontakte zwischen Europāern und Inselbewohnern bestanden. Vgl. Anja Schwarz' "Linien im Sand. Der Sudseestrand als Begegnungsraum bei James Cook und Ceorg Forster $\%$, in: Zeitschrift für Kulturwissenschaften 2 (2008), S. 53-64, hier: S. 53. 
lich threr jeweiligen Waflensysteme besteht, nicht ausnutzen. sondern bemüht sind, sich den Einheimischen als friedliche Gäste darzustellen.

Nachdem das Moment der ersten Unsicherheit überwunden ist. kommt es in einem zweiten Schritt auf beiden Seiten zu dem Versuch. vertraute Kommunikationsformen für die neue Situation zu nutzen. Gleichzeitig herrscht jedoch bei beiden Parteien das Bewusstsein, dass die andere Seite möglicherweise nicht alles verstehen wird. Insofern ist der Versuch, sich gegenseitig kennen zu lernen, ein äußerst komplexer Prozess; Auf der einen Seite haben beide Seiten die Tendenz, so matürlich. wie möglich (d. h. unter Belbehaltung möglichst vieler vertrauler Verhaltensmuster) auf die fremden Menschen zu reagieren. ${ }^{3}$ Auf der anderen Seite verschiebt die schnell offenbar werdende Schwierigkeit, sich auf gewohnte Weise verstăndlich zu machen, die Grenzen dessen, was bis dahin als selbstverständlich gelten mochte. Der Lemprozess, der bei beiden in Gang gesetzt wird, verdankt sich einer permanenten Rückkoppelung zwischen eigenem und fremden Verhalten, einer Rückkoppelung, die jedoch nur so lange funktioniert, wie nicht als fundamental erlebte, unhintergehbare Werte durch das Neue in Frage gestellt werden. Und genau in einem solchen Moment, in dem die Anpassung an den jeweils anderen an eine Grenze stößt, beginnen die eigentlichen Schwierigkeiten der Kommunikation.

Die Europäer stehen anfangs in der Erwartung, dass ihnen die Lundung auf den Inseln verwehrt werden könnte. Ausgehend von europăischen Begriffen, betrachten sie die Inseln als Musterbeispiele für ein klar abgegrenztes Territorium. Da, wo das Wasser ans Land stößt, beginnen die Besitzansprüche derer, die das Land bewohnen: die Ansprüche einer Nation. Die Frage, die sich für Kontinente oder ausgedehnte Landstriche stellt, die Frage nâmlich, wie das Gebiet in den Augen derer, die es bewohnen, gegliedert ist und wo wessen Besilzansprüche überhaupt beginnen. hat für Inseln keine Relevanz. ${ }^{4}$ Thre Überschaubarkeit legitimiert in den Augen der

3 Tzvetan Todorov zufolge ist diese Reaktion auch fur den Kontakt zwischen Europaern und Kulturen Sudamerikas von großer Bedeutung gewesen. Die Azteken seien ihrer traditionellen Symbolik gefolgt, wahrend es die Spanier verstanden hatten, schon fruhzeitig neue Verhaltensweisen jenseits ihres rituellen Verstandnisses zu entwickein. Dies hat das machtpolitische Ungleichgewicht erzeugt, aus dem die Zerstorung aztekischer Lebensformen resultierte. Vgl. Tzvetan Todorov: La conquéte de l'Amérique. La question de l'autre, Paris: Seuil 1982

4 Dies ist bereits als kolonialer Diskurs zu kennzeichnen: Das Land, das noch in niemandes Besitz sei, wird ais ungenutztes Land betrachtet, das man jederzeit in Besitz nehmen konne. Wie diese Inbesitznahme genau erfolgt, wird im Folgenden gezeigr werden. 
Europāer die Idee, dass Inseln eine in sich abgeschlossene Einheit bilden und mithin nur ihr eigener, erster Schritt ans Land entscheidend ist: Sobald sie thren Fuß auf das Land setzen dürfen, haben sie gleichsam keine weiteren Grenzüberschreitungen durchzusetzen. sondern sind da, wo sie sind: auf einer Insel, auf die auch sle Anspruch erheben dürfen. Mit diesen Ansprüchen aber verhălt es sich so, dass sie in eben dem Maße wachsen, in dem ihnen keine Grenzen gesetzt werden. Dies wird im Folgenden zu zeigen sein.

III.

Die Schwierigkeit für die Reisenden unter Cook besteht darin, dass es auf der Insel selbst Orte gibt, die, obwohl zum Territorium der Insel gehörend, den Inselbewohnern als Grenzgebiet gelten. Bestimmte Begräbnisorte. Wohnstätten hochgestellter Personen oder Plātze, an denen - ökonomisch besonders wertvolle - Schweine gehalten werden, sollen den Europäern verschlossen bleiben. ${ }^{5}$ Immer wieder erweist sich jedoch, dass die Europāer das, was nach thren eigenen Kategorien überraschend sein müsste - ihr ungehinderter Zugang zu einem Territorium, das sich im Besitz fremder Menschen befindet -, schnell als selbstverstândlich wahrnehmen. Aus der Leichtigkeit, mit der ihnen die Landung auf den Inseln im allgemelnen gestattet wird, schlleßen sie auf ihr Recht, die gesamte Insel in Augenschein zu nehmen. Aus der fehlenden Grenzziehung durch die Inselbewohner folgt also nicht ein besonderer Respekt für die intemen Grenzziehungen, die von Seiten der Einheimischen als notwendig betrachtet werden. Im Gegenteil: Legitimiert durch wissenschaftliche Interessen ${ }^{6}$ und die Notwendigkeit zur Versorgung mit Lebensmitteln, sind es die Ankömmlinge, die ihre eigenen Vorstellungen von 'Grenze' durchzusetzen versuchen. Dafür steht beispielhaft die folgende Mahlzeit, die die Europäer auf Tahitt einnehmen:

»Nachdem wir etiche Meilen weit gegangen waren, setzten wir uns auf einige große Steine nieder, die vor einer Hutte eine Art von erhohtem Pflaster ausmachten, und bathen die Einwohner, daß sie uns, gegen baare Zahlung in Co-

5 Wichtig ist an dieser Stelle, dass bestimmte Orte auch fü die Einheimischen mit einem Tabu belegt sind. Das Verbot betrifft also durchaus nicht nur die Europaer.

6 Eine wissenschaftsgeschichtliche Publikation zur Dialektik der Săkularisierung in Ceorg Forsters Reise um die Welt ist in Vorbereitung. Vgl. Anne D. Peiter: "Entre sciences et merveilles. Les méduses dans le récit, Voyage autours du monder de Georg Forster, erscheint voraussichtlich in einem vom Forschungszentrum CRLHOI herausgegebenen Band zum Indischen Ozean. 
rallen, etwas. Brodfrucht und Coco-Nüsse verschaffen mogten. Sie waren sehr willig dazu, brachten herbey was sie hatten und in der Geschwindigkeit stand das Frühstück aufgetischt vor uns. Um es desto ruhiger zu verzehren, ließen wir den ganzen Haufen unserer Begleiter in einiger Entfernung von uns niedersitzen, damit sie keine Gelegenheit haben mogten, Gewehr oder andre Dinge zu erhaschen, die wir beym Essen von uns legen mussten. «?

Hier werden Mitglieder von Cooks Besatzung auf das Schōnste mit Nahrung versorgt. Zwar erfolgt eine Bezahlung für das Frühstück, dennoch trägt die gesamte Szene paradiesische Züge. Das, was an Bezahlung für die Früchte zu entrichten ist, stellt nämlich in den Augen der Europäer keinen wirklichen Wert dar: Sie bezahlen mit Glaskorallen, einem Produkt, das nur dadurch Wert für sie hat, dass die Inselbewohner es als wertvoll betrachten. Während Eisenwerkzeuge und Nägel auch in den Augen der Europäer als nützlich und daher (in gewissen Grenzen) als wertvoll anerkannt werden, sind die Glaskorallen allein aus praktischen Gründen mit auf die Reise genommen worden: Sie sind billig herzustellen und den Inselbewohnern vom Material her unbekannt. Das Neuartige aber sichert das Interesse an ihnen. Außerdem können diese "falschen" Korallen leicht in großen Mengen transportiert werden, ohne das Gewicht der Schiffsladung übermäßig zu steigern. Ein weiterer Vorteil besteht darin, dass sie - darin in der Tat Geldmünzen ähnlích -, in kleinen Einheiten gegen Dienste und fremde Produkte eingetauscht werden kŏnnen, dass es also keines gleichartĩgen -Wechselgeldes" bedarf. um etwas zu kaufen.

Wenn man nun davon ausgeht, dass sich das wundersam prompt aufgetischte Mahl den Europäern als große Wohltat darstellt, muss auffallen, dass sie dieses zur Ziehung von Grenzen und nicht etwa zu deren Einebnung nutzen: Die Inselbewohner werden nicht mit zu Tisch gebeten, sondern angewiesen, sich in einiger Entfernung von den Essenden niederzusetzen. Elias Canetti vertritt in seiner poetischen Anthropologie. Masse und Macht die These, dass es stets die Machthaber seien, die versuchten, freien Raum um sich zu schaffen:

Jeder, auch der niedrigste, sucht zu verhindern, dass man ihm zu nahe kommt. Wo immer eine Form des Zusammenlebens sich zwischen Menschen etabliert hat, druckt sie sich in Abstanden aus, die ihnen diese unablässige Angst des Gepackt- und Ergriffenwerdens benehmen. [...] Der Machthaber, von

7 Georg Forster: Reise um die Welt, Frankfurt/Main: Insel Verlag 2004, 5. 269 (Hervorhebung A.P.). 
dessen Existenz die der ubrigen abhängt, erfreut sich des größten, des deutlichsten Abstands (... J, , $^{\text {B }}$

Canetti betrachtet das Bedürfnis nach Distanz als etne quasi tierische Verhaltensweise, die mit der Angst, zur Beute der anderen zu werden, zusammenhânge. Und in der Tat spiegelt sich in Georg Forsters Text die Sorge, die Waffen, die die Europäer bei ihren ersten Erkundungen Tahitis stets mit sich führen, könnten in die Hände der Einheimischen gelangen und sich so gegen sie, die Ankömmlinge, wenden. Von der Überlegenheit der europälschen Waffen hängt jedoch die Aufrechterhaltung der Asymmetrie, die auch das Essen dokumentiert, wesentlich ab. Während die Inselbewohner es also zugelassen hatten, dass die Reisenden den freien Raum, der zwischen thnen lag, immer weiter reduzierten - man könnte sagen, sie rückten den Einheimischen regelrecht auf die Pelle -, demonstrieren die Europãer mit ihrer nur scheinbar unscheinbaren Geste hier sitzen wir, dort ihr, dass sie sich als potentielle Beherrscher der Insel sehen.

\section{IV.}

Aus einer spâteren Situation wird ersichtlich, dass die Distanz zwischen beiden Gruppen regelmäßig ins Werk gesetzt wird, jetzt jedoch mit einer entscheidenden Änderung:

wWir versorgten uns [...] mit einem hinlänglichen Vorrath und mietheten einige Leute um uns das Eingekaufte nachtragen zu lassen. Nachdem wir ohngefähr funf englische Meilen weit gegangen waren, setzten wir uns auf einen schönen Rasen unter den Baumen nieder, um Mittag zu halten. Nachst den unterwegens angeschaften Früchten bestand unsre Mahizeit aus etwas Schweinefleisch und Fischen, welche wir vom Bord mitgenommen hatten. Die Tahitier machten einen Creis um uns her, unsern Wegweisern und Helfern aber gaben wir die Erlaubniß, sich neben uns zu setzen. Sie ließen sich's herzlich gut schmecken [...]. $x^{9}$

Wieder geht es um Essen, wieder ist die Rede von einer beschaulich-idyllischen Situation, die sich nicht zuletzt der Schönheit der Natur verdankt. Als Wiederholung erscheint auch die râumliche Ordnung, dic auf Befehl der Europäer zustande kommt: Während das ,Wir der Europāer ins Zentrum rückt, werden die Tahitier - das Ihr' - auf eine Weise platziert. dass eine Grenze entsteht. Erneut

8 Elias Canetti: Masse und Macht, Frankfurt/Main Fischer TaschenbuchVerlag 2001, S. 242 (Hervorhebungen A. P.),

9 G. Forster: Reise um die Welt, S. 312 (Hervorhebung A. P.). 
geraten die Einheimischen, die doch bisher die Akteure der Insel waren, in die Rolle von Zuschauern. Das Sitzen im Kreis bedeutet zugleich, dass sie nicht die gleiche räumliche Konzentration aufweisen wie die Reisenden. Diese sitzen zusammen, in körperlichem Kontakt miteinander, könnten sich, wenn der Raum, den sie im wahrsten Sinne des Wortes in Be-Sitz nehmen, mit ihren Waffen verteidigen. Und doch ist die Situation wesentlich von der vorherigen verschieden: Die Trennung von Wir und Ihr hat an Schârfe verloren. Einige Tahitier werden als zugehörig betrachtet. Sie bleiben zwar diejenigen, die gemietet, und zeitweilig als Besitz betrachtet werden dürten, doch durch thre Funktionen werden sie von den Europäern in gewisser Weise als gleichberechtigt anerkannt, Die Gleichberechtigung drückt sich darin aus, dass sie mit den neuen Be-sitzern sitzen dürfen und so von den übrigen Einheimischen abgesondert werden.

Indem das Prinzip des divide et impera zur Anwendung kommt. verschärft sich jedoch die Grenzziehung zwischen beiden Gruppen. Die Beobachtung, dass ein Teil der Tahitier die ,Fronten wechselt. und mit den Europāern speisen darf, scheint dieser Behauptung entgegenzustehen. Doch ein Vergleich zu der vorhergegangen Situation erweist ihre Berechtigung: Während die Europäer dort allein auf ihre Waften vertrauen konnten, haben sie hier Verbündete gewonnen, die einen doppelten Schutz für die Grenze bedeuten, die sich zwischen beiden Gruppen etabliert hat. Denn so sehr auch zuzugeben ist. dass sich mit einigen Tahitiern ein reger Austausch entspinnt, so sehr bleibt doch festzuhalten. dass die Europäer diese Kontakte zur Aufrechterhaltung ihrer Dominanz benutzen.

Das Zusammensitzen und -speisen mit ausgewählten Inselbewohnern sind von Vorteil für sie: Sie werden genảhrt, aber zugleich ist es doch, als wâren sie selbst es, die năhrten. Ohne die Bereitschaft, ausgewāhlte Fremde im Zentrum, dem Platż der Europäer. zuzulassen, blieben sämtliche Einheimischen in der Rolle der $\mathbf{Z u}$ schauer des Mahls. So aber wird Nahrung, wenigstens teilweise, geteilt - die Geste scheinbarer Großzügigkeit geht von den Europäern aus und nicht etwa von denjenigen, die die Nahrung überhaupt besorgten. ${ }^{10}$ Denn diese Besorgung ist. sobald das Essen beginnt.

T0 Hierbei ist allerdings zu bedenken, dass das Dokument, auf das ich mich stutze, von einem Europäer geschrieben ist. Er schildert seine Sichtweise, die zudem eine retrospektive ist: Der Ausgang der Begegnung, eine gewisse Dominanz der Europaer, scheint fur ihn klar zutage zu liegen. Und so verwischt Forster vielleicht unbewusst Erlebnisse, die das Gegenteil hátten zeigen kónnen. - Mit dieser Frage hangt auch die Debatte zwischen Marshall Sahlins und Gananath Obeyesekere uber die Wahrnehmung Captain Cooks durch die Bevolkerung auf Hawail und die Umstande seines Todes zusammen. Ist die Vergottlichung Cooks eine Realitat gewesen oder muss 
längst in Vergessenheit geraten. Jetzt zählt nur noch das Essen selbst. Canetti stellt in diesem Zusammenhang die provozierende These einer Ähnlichkeit zum Tierreich auf: $\amalg$

wSchwer und voller Behagen liegt er da, der am meisten fressen kann, dèr Meistesser. Es gibt Gruppen von Menschen, die in einem solchen Meistesser ihren Hảuptling sehen. Sein immer gestillter Appetit erscheint ihnen als Gewähr dafur, dass sie selber nie lange Hunger leiden werden. Sie verlassen sich auf seinen gefülten Bauch, als hătte er ihn für sie alle mitgefülit. Der Zusammenhang von Verdauung und Macht tritt hier klar zutage.«12

Canetti formuliert, was in ähnlicher Weise auch auf das von den Tahitiern zur Verfügung gestellte, von den Europäern verzehrte und nur zum Teil an die Produzenten der Nahrung weitergereichte Mahl zutrifft: Der Umstand, dass nicht alle Tahitier zu essen bekommen, scheint dadurch wettgemacht zu werden, dass es sich die Europãer schmecken lassen. Diese Wahrnehmung ist durchaus nicht nur die

man sie als bloßen europáischen Mythos betrachten, wie Obeyesekere in seiner Kritik an der Quelleninterpretation Sahlins das tut? Trug Cook selbst durch sein Fehlverhalten Verantwortung für die Gewalt, die ihn um sein Leben brachte, oder wurde er Opfer eines Ritualmordes? Grundsătzlicher formuliert: Macht Sahlins die Hawaiianer auf eurozentrische Weise zu Marionetten ihrer kulturellen Muster, indem er sie fur irrational erklärt, oder unterliegt umgekehrt Obeyesekere der Versuchung, den Hawailanern eine westliche Rationalitat zuzuschreiben und damit selbst ethnozentrisch zu argumentieren? Der Ansatz Sahlins, Kulturkontakte aus der Perspektive der indigenen Bevölkerung des Pazifik zu beschreiben, ist in jedem Fall für die -Kulturalisierung der Ethnologie von großer Bedeutung gewesen und trifft sich daher mit der kulturwissenschaftlichen Analyse, die ich selbst versuche. Sahlins kritisiert Obeyesekere mit folgendem Argument: wf the underlying argument is that all nativest are alike, the superimposed argument is that they one and all enjoy a healthy, pragmatic, flexible, rational, and instrumental relation to the empirical realities. Reflecting rationally (and transparently) on sensory experience, they are able to know things as they truly are. V Vgl. die Kritik an Sahlins durch Gananath Obeyesekere: The Apotheosis of Captain Cook. European Mythmaking in the Pacific, Princeton. Princeton UP 1992, sowie in Reaktion darauf: Marshall Sahlins: How $\bowtie$ Natives $\approx$ think. About Captain Cook, for example, Chicago, London: University of Chicago Press 1995, besonders S. 85-117; das obige Zitat auf S. 5.

11 Vgl, dazu: Anne D. Peiter: Komik und Gewalt. Zur literarischen Verarbeitung der beiden Weltkriege und der Shoah, Köln u. a.: Bohlau 2007, besonders Kapitel 8. Außerdem dies. "Der Mensch als Tier, das Tier als Mensch? Die Bedeutung von Natur für Elias Canettis Analyse der Shoahs, in: Hubert Zapf ( $\mathrm{Hg.}$.), Kulturökologie und Literatur. Beitràge zu einem transdisziplinären Paradigma der Literaturwissenschaft, (Anglistische Forschungen, Bd, 387). Heidelberg: Winter 2008, S. 229-240.

12 E. Canetti: Masse und Macht, S. 257. 
Wahrnehmung derjenigen, für die das Mahl überhaupt erst gerichtet wurde, nämlich der Europäer. Vielmehr scheinen auch die Tahitier. die zur Teilnahme eingeladen sind, darauf zu vertrauen, dass das Essen der Europäer und das Essen für sie selbst nit einem Essen für alle gleichzusetzen ist. Die Teilhabe an Privilegien ist derart verlockend, dass in Vergessenheit gerät, dass die Inselbewohner thre Nahrung zuvor nicht zu teilen hatten. Obwohl also vor der Ankunft der Europäer mehr Nahrung vorhanden war als jetzt, wird der Anspruch derselben auf Nahrung als Zeichen ihrer Überlegenheit (und nicht ihrer Abhängigkeit) gewertet. ${ }^{\text {ld }}$

\section{V.}

Wie sehr die Grenzziehung. die durch die Teilung der Kräfte der Einheimischen an Dauerhaftigkeit gewinnt, auf Seiten der Europäer zu einem allgemeinen Prinzip der Kommunikation erhoben wird. geht daraus hervor. dass sie auch auf anderen, weit entfernten Insein - nämlich den neuen Hebrideninseln - zur Anwendung kommt:

-Der Anschein ließ uns eine ganz ruhige Landung hoffen, denn die Zahi der an der Kuste befindlichen Einwohner war zu gering, um uns dieselbe streitig zu machen. Sie hatten sich, nicht weit von der See, ins Gras gelagert, und liefen auch wurklich fort, als sie uns aus dem Boote steigen sahen; da wir ihnen aber freundlich zuwinkten, so kehrten sie wieder zuruck. Von Westen kam ein Haufe von etwa 150 Wilden her, die allesammt, in der einen Hand Waffen, in der andern aber grune Palmenzweige trugen. Diese uberreichten sie uns als Friedenszeichen, und wir beschenkten sie dagegen mit Medaillen, Tahitischem Zeug und Eisenwerk, tauschten auch für dergleichen Waaren etliche Cocosnusse ein, nachdem es eine ganze Weile gedauert hatte, ehe sie, aus unserem Hindeuten auf die Cocos-Palmen und aus andern Gebehren, begreifen Konnten, dass wir von diesen Bäumen die Fruchte zu haben wunschten. Hierauf verlangten wir, dass sie sich alle niedersetzen mogten, welches auch zum Theil geschahe, und alsdann ward ihnen angedeutet, dass sie eine in den Sand gezogene Linie nicht uberschreiten sollten, womit sie ebenfalls zufrieden waren. $\alpha^{14}$

Noch einmal geht es um den Beginn einer Kontaktaufnahme. Neu Ist an dieser Situation, dass die Bewaffnung von den Inselbewohnern zur Schau gestellt wird. Dass die Europäer ihre eigenen Waffen zurûckhalten, sie weder vorzeigen, noch benutzen, verdankt sich

13 Diese Form der „Kollaboration* steht wahrscheinlich im Zusammenhang mit den Machtstrukturen, die auf der Insel selbst herrschten. Die Machttechniken def Europàer konnten also in gewisser Weise als vertraute interpretiert werden.

14 G. Forster Reise um die Welt, S. 729f. (Hervorhebung A. P.). 
aber offenbar nicht der Entscheidung, prinzipiell Verzicht auf sie zu tun, sondern dem Bewusstsein, dass ihre Uberlegenheit obnehin nicht in Frage steht: Es heißt, die Einwohner könnten aufgrund ihrer geringen Zahl den Ankômmlingen die Insel nicht sstreitig machen.. Die Überlegenheit der europäischen Streiter sieht man nicht zuletzt daran, dass, sobald die Nahrungssorgen beseitigt sind, der hinreichend bekannte Versuch unternommen wird, die zu den belden Gruppen gehörenden Kôrper auf eine bestimmte Weise im Raum anzuordnen. Anders als zuvor wird die Grenze jedoch nicht mehr nur durch die Körper selbst gekennzeichnet. Vielmehr tritt eine Linie, die in den Sand gezogen wird, hinzu. ${ }^{15}$

Während Körper beweglich sind und unterschiedliche Positionen im Raum einnehmen können, ist die Grenzlinie von einer gewissen Dauer. Der Umstand, dass die Einwohner sich erst setzen müssen, bevor die Grenze gezogen wird, deutet auf die eingeschränkte Beweglichkeit hin, die den Einheimischen auferlegt werden soll. Wenn sie stünden, kỏnnten sie die Grenzziehung leichter verhindem. Dass nur diejenigen, die die Grenze ziehen, stehen und gehen, der Großteil der Einheimischen hingegen sizzt, verdankt sich also einem strategischen Kalkül:

„Eine gewisse Distanz der anderen um einen herum macht mehr aus dem Stehenden. Einer, der allein, durch eine Art von Entfernung getrennt, vielen anderen gegenubersteht, wirkt besonders groß, so als stünde er für sie alle zusammen allein. 16 $^{16}$

Gilt die gesteigerte Wahrnehmung von Grôße schon für den Stehenden. der Distanz zu den anderen beansprucht - wie viel mehr gilt diese Beobachtung erst, wenn die anderen sitzen, d, h, sich kleiner fühlen mūssen, als síe in Wirklichkeit sind. Dass der Eingriff in die Bewegungen der Körper wirkliche machtpolitische Konsequenzen hat, geht aus dem hervor, was der Grenzziehung folgt:

"Wahrend dieser Zeit hatten die Soldaten sich in Ordnung gestellt, und die indianer bezeigten so viel Furcht fur ihnen, dass sie, bey der geringsten Bewegung derselben, allemal eine Ecke fortliefen; nur etliche alte Männer waren so herzhaft sich dadurch nicht erschrecken zu lassen. Wir verlangten, dass sie thre Waffen von sich legen sollten, welcher Forderung, so unbillig sie an sich auch seyn mochte, dennoch von den mehresten Genuge geleistet wurde, in 17

$15 \mathrm{Vgl}$. dazu erneut Anja Schwarz, die auf die sprichwötliche Bedeutung des englischen Ausdrucks „draw a line in the sand aufmerksam macht: Er bedeute s[to establish] a limit or boundary: [to specify] a level of tolerance or

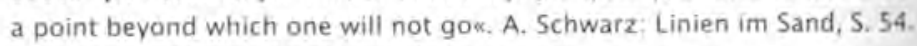

16 E. Canetti Masse und Macht, S. 460.

17 G. Forster: Reise um die Welt, S. 730. 
Georg Forster ist selbstkritisch genug, um zuzugeben, dass die Forderung der Europäer unakzeptabel sein musste. Der Erfolg verleitet aber auch thn dazu. die Sicherung der eigenen Überlegenheit schließlich zu legitimieren. Die Notwendigkeit dieser Maßnahmen wird durch den fehlenden Respekt der Inselbewohner für das Eigentum der Europãer begründet. Bei einem Tauschhandel kommt es nâmlich zu einem Konflikt zwischen Captain Cook und einem Inselbewohner. Cook erwartet im Tausch für eine tahitische Kleidung. die er dem anderen bereits ausgehändigt hat, eine Keule, die er aber plötzlich nicht erhält. Daraufhin schießt er dem "Betrüger* eine Ladung Schrot ins Gesicht. ${ }^{i *}$ Der Konflikt eskaliert. trotz der Bemūhungen eines alten Inselbewohners, der durch das Angebot von Nahrung zwischen beiden Parteien zu vermitteln versucht. Nachdem die Europäer ihre gefährlichsten Waffen, vor allem die Kanone, zum Einsatz gebracht haben, entscheiden sie sich, ihre Grenzziehung zu verschärfen:

-Wir ließen es nunmehro unsre erste Sorge seyn, zu Bedeckung der Arbeitsleute, die See-Soldaten in zwo Linien zu stellen. An beyden Seiten schlug man Pfahle in die Erde, und zog einen Strick dazwischen, so dass die Wasserschöpfer einen Platz von wenigstens 150 Fus breit inne hatten, wo sie ihre Arbeit ohngestört vornehmen konnten. Nach und nach kamen die Einwohner, aus dem Gebusch, auf den Strand. wir winkten ihnen aber jenseirs unsrer Linien zu bleiben, welches sie auch allerseits beobachteten. Der Capitain wiederhoite nur seine vorige Zumuthung, dass sie ihre Waffen niederlegen mogten. Der größere Haufer, an der Westseite, kehrte sich nicht daran; die andere Parthey hingegen, die mit dem friedlichen Aiten einerley Sinnes zu seyn schien, ließ sich großtentheils dazu bewegen. Diesem Alten, der Pao-vjangom hieß, hatten wir, als Beweis unsers Zutrauens, vorzugsweise die Erlaubniß gegeben, sich innerhalb der abgesteckten Linien aufhalten zu durfen. ${ }^{19}$

Hier sieht man, wie die Grenzziehung immer deutlichere Formen annimmt: Die Linie, die bis dahin, materiell kaum sichtbar, in den Sand gemalt worden war. verliert ihren provisorischen Charakter. Plöcke und Strick sind nicht verwischbar wie die Linie, nehmen Dauerhaftigkeit für sich in Anspruch. Die Grenze wächst in die Höhe, richtet sich auf: Die horizontale Linie aus Sand wird ergänzt durch vertikale Markierungen. die die Europäer scharf von den

$18 \mathrm{Vgl}$. ebd., S. 733.

19 Ebd., S. 735f. (Hervorhebungen A. P.) - Genau diese Passage in Forsters Reisebericht wird auch von Schwarz interpretiert: "An diesem ersten gemeinsamen Tag am Strand, so scheint es, ist es die Sichtbarkeit und gegenseitige Anerkennung der Linien, welche das offen zur Schau getragene Aggressionspotential beider Seiten in Schach hält. [...] Dank der Markierungen gehen Einheimische und Fremde nun jeweils auf ihrer Seite der Linie dem Tagesgeschaft nacha. A. Schwarz, Linien im Sand, 5. 58. 
Einheimischen scheiden. Dass der als friedlich eingestufte Alte die Grenze überschreiten darf, zeigt paradoxerweise, dass sich die Europäer in Schwierigkeiten befinden. Es ist leichter für sie, unter friedlichen Bedingungen Vorräte anzulegen - insbesondere das Schiff mit Wasser zu versorgen -, als unter der Drohung, angegriffen zu werden. Insofern ist die Aufweichung der Grenze als Versuch zu deuten, die Inselbewohner zu einer Rückkehr zur Ausgangssituation zu bewegen.

Wie wir gesehen haben, hatten diese bei der Begrüßung der Europäer zwei Gegenstände vorgezeigt: ihre Waffen (als Zeichen ihrer Wehrhaftigkeit) und Palmenzweige (als Zetchen ihrer Bereitschaft zu einem friedlichen Austausch). Wenn jetzt die Waffen dominieren, erweist sich die Strategie der Europãer, nach Überschretung der Insel-Grenze den anderen unverzüglich Grenzen aufzuerlegen, als falsch. Es wird nămlich deutlich, dass, so sehr auch europāische Linien die Insel ûberziehen mōgen, die Einwohnerschaft threrseits klare Vorstellungen von den Grenzen hat, die von Seiten der Europãer nicht ūberschritten werden dürfen. Der Einsatz von kỏrperlicher Gewalt gehört zu dieser Grenzüberschreitung. ${ }^{20}$ Sie ist den Bewohnern dieser Insel ebenso unverständlich wie denen der Nachbarinseln, weil offenbar die europäische Konzeption von Besitz (Unterschlagung des Tauschobjektes bei einem Handel) nicht die gleiche ist. Dass die Grenzüberschreitung der Europãer dann jedoch beim Schrot nicht Halt macht, sondern im Gegenteil die Ûberschreitung durch eine unverhälnnismäßige Fortsetzung der Ụberschreitung noch übertrifft, zeugt von dem Willen der Reisenden, ihren Grenzvorstellungen um jeden Preis zur Durchsetzung zu verhelfen. Der oben angedeutete Konflikt eskaliert auf folgende Wefse:

sUm sie also, wo möglich, im Voraus davon [einem Angriff namlich, A. P.] abzuschrecken, ließ Captain Cook eine Flintenkugel uber ihre Kopfe hinfeuern. Der unvermuthete Knall brachte auch wurklich den ganzen Haufen in Bewegung, so bald aber das erste Erstaunen vorüber war, blieben sie fast alle wieder stehen Einer, der dicht ans Ufer kam, hatte sogar die Verwegenheit, uns den Hintern zu zeigen und mit der Hand darauf zu klatschen, weiches, unter allen Volkern im Süd-Meer, das gewöhnliche Zeichen der Herausforderung ist. Dies Großsprechers wegen ließ der Capitain noch einen Flintenschuss in die Luft thun; und da man dieses auf dem Schiffe fur ein Signal hielt, so ward alles grobe Geschutz, welches aus 5 vierpfundigen Kanonen, zwey halbpfundigen DrehBassen, und vier Musketons bestand, mit einem male abgefeuert. Die Kugein pfiffen uber die indianer weg, und kappten etliche Palmenbaume; dadurch er-

20 Die Grenze ist nicht nur etwas Raumliches, sondern bezieht sich auch auf die Verhaltensweisen der Fremden. 
reichten wir unsern Zweck, dass nemlich in wenig Augenblicken nicht ein Mann mehr auf dem Strande zu sehen war. 21

Hier steht eine symbolische Waffe (der Hintern) der echten Waffe (der Flinte. gleich darauf den Kanonen und Musketen) gegenüber. Der Zweck, den die Europäer anstreben. wird von Forster als erreicht bezeichnet: Der Strand leert sich im wahrsten Sinne des Wortes ischlagartig. Dem Schlag auf den Hintern. der (wohl auch weil er nah am Ufer erfolgt) als Herausforderung wahrgenommen wird, antwortet der Schlag der Geschosse, die an den Palmen das exerzieren, was, bei einer Fortsetzung des Konflikts, auch den Menschen drohen würde: Die Köpfe der Bäume werden sgekappt*. Durch diese Demonstration der europäischen Übermacht wird also die Grenze zwischen Wasser und Land - der Strand - für die Europäer ageräumts. Und als dann die Inselbewohner zurückkehren, übernehmen die beschriebenen Pfähle und Seile die Aufgabe, daran zu erinnern, dass der Strand ohne weiteres wieder zum alleinigen Territorium der Europäer gemacht werden könnte.

Diese neue Grenze verurteilt die Inselbewohner dazu, ihre Bewegungen zu kontrollieren, um nicht in die verbotene Zone einzudringen. Galt den Europäern die Bewegungslosigkeit, zu der die Angegriffenen nach der ersten Flintenkugel fanden, aufgrund des Mutes, von dem sie zeugte, als Provokation, so wird jetzt die Kontrolle über die Bewegungsabläufe als notwendiger Beweis für die Fähigkeit der Europäer gewertet, über die Richtung und Art der Bewegung der Fremden dauerhaft zu bestimmen. Die Grenze ist damit nicht nur etwas, wodurch die Insel selbst gegliedert und aufgeteilt wird, sondern vielmehr etwas, was das Verhāltnis, das für die Einheimischen bisher zwischen ihren eigenen Körpern und dem vertrauten Raum bestand, von Grund auf verändert.

Dass die Europâer das Hintern-Zeigen mit Musketenschüssen beantworten, das Aufs-Gesäß-Klopfen mit Kanonendonner, nimmt sich aus wie ein Übermaß an Ernsthaftigkeit. Obwohl das Ungleichgewicht zwischen herausfordernd präsentiertem. doch unbewaffnetem Körper auf der einen und technisch avanciertem Waffenarsenal auf der anderen Seite offen zu tage liegt, muss die Überlegenheit in den Augen der Europäer in die Tat umgesetzt werden. Die Zeichenhaftigkeit der Körper reicht nicht aus, denn sie vermeidet, dass die Körper sich berühren: Der vorgezeigte Hintern schießt nicht, er zeigt sich nur. Die Walfen der Europäer haben hingegen die Eigenschaft, die Körper treffen zu können, ohne deshalb die geforderte Distanz aufgeben zu müssen. Die Distanzen, die allein von den Kugeln úberwunden werden, übertreffen alles, was die Waflen der Inselbe-

21 G. Forster: Reise um die Weit, S, 735 (Hervorhebung A. P.). 
wohner zu leisten vermögen. Gerade darum zerstören sie den komischen Aspekt, der der Geste des Einheimischen anhaftete. Die Geste mochte besagen: Kommt doch näher, wenn ihr könnt, Ich verlache Euch, statt Euch zu fürchten, Indem die Europäer als Antwort ihre Kugeln schicken, heben sie die Distanz, die der Provokateur durch die Komik zwischen sich und den Eindringlingen aufgerichtet hatte, auf. Auf die Distanzierung, die das Lachen leistet, folgt keineswegs der Versuch, die Inselbewohner ihrerseits als lachhaft hinzustellen. Vielmehr beenden die Kugeln den soeben erst begonnenen symbolischen Streit und machen ihn zu einer Sache, in der es allen Ernstes um Leben und Tod geht. In Vergessenheit gerät dabei auf Seiten der Europäer, dass die provozierende Geste des Auf-denHintern-Klatschens eine Antwort auf eine Schrotladung darstellte, die einem anderen Inselbewohner ins Gesicht fuhr.

IV.

Die Komik als Verzicht auf direkte Gewalt ist in noch einer Szene greifbar. Sie spielt nach einem von Einheimischen und Europảem gemeinsam unternommenen Austlug, der durch ein Leck alle in Gefahr gebracht hatte.

»Am folgenden Morgen machten sich die Matrosen an die Ausbesserung des Boots, und ließen ihre nassen Kleider in der Sonne trocknen. Die Indianer versammelten sich aus allen Gegenden der Insel in solcher Anzahl um sie her, dass Herr Pickersgill, zu Sicherung der Kleider, fur nöthig fand, Linien in den Sand zu ziehen, die keiner von den Wilden uberschreiten sollte. Sie begriffen, was diese Verfugung sagen wollte, und ließen sich solche ohne Widerrede oder Widerspenstigkeit gefallen. Und dem ganzen Haufen war nur Einer, der uber diese Anstalt mehr Verwunderung, als die ubrigen, bezeugte, und eben dieser fieng, nach einer Weile, sehr launigt, an, mit einem Stock einen Kreis um sich herzuziehn, und unter allerhand possierlichen Grimassen den Anwesenden zu verstehen zu geben, daß sie auch ihm vom Leibe bleiben soliten. Bey der sonst gewöhnlichen Ernsthaftigkeit der Einwohner, war dieser humorose Einfall sonderbar und merkwürdig genugl $\varkappa^{22}$

Wir begegnen hier den schon vertrauten Kommunikationsformen der Europäer. Sie schaffen Distanz, indem sie mit Linien die Grenzen ihres Körpers gleichsam erweitern und den Fremden zu verstehen geben, dass sie eine Annäherung über diese Linie hinaus nicht wünschen. Ihre Körper werden immer platzgreifender.

Die Subversion des Mannes, der, anders als die anderen, das Ungeheuerliche dieser Regelung nicht beiseite schieben mag, be

22 Ebd., 5. 855 (Hervorhebungen A. P.). 
steht in der Wiederholung. Er tut nichts anderes, als das, was er beobachtet hat, nachzuahmen. ${ }^{23}$ Indem er sich also als der eifrigste Schüler der Europäer gebärdet, sorgt er paradoxerweise erst für die Sichtbarkeit dessen, was von den anderen sogleich in eine - wenngleich neuartige - Normalität integriert zu werden drohte: Er zeigt. dass Linien, die zwei Menschengruppen gegeneinander abgrenzen. durchaus nicht selbstverständlich sind. Dass sie nicht selbstverståndlich sind, wird dadurch demonstriert. dass er, wie die Europäer, das Recht für sich in Anspruch nimmt, auf der Insel weitere kleine Inseln zu bilden, Inseln, die bestimmten Kategorien von Menschen - nämlich ihm selbst - vorbehalten sind.

Die komische Qualität seines Spiels besteht darin, dass er die Tendenz. die der von den Europäern installierten Raumordnung innewohnt. ins Extrem treibt. Die Europäer hatten zwei Gruppen unterschieden: eine. die von den Mitgliedern der europăischen Besatzung gebildet wurde. und eine. die als fremd und potentiell feindlich aus dieser ausgegrenzt wurde. Dadurch, dass sich diese Aufteilung bei der Landung auf den verschiedensten Inseln wiederholte, entstand der Eindruck eines Solipsismus. Dieser lebt von übersteigerten Grenzziehungen. Wenn nämlich auf einer Reise das Fremde stets aus dem herausgehalten wird, was (obwohl dem Fremden gehörend) als Territorium des Eigenen beansprucht wird, schwindet der Austausch. Und mit der Einschränkung des Austauschs schwindet zugleich auch die Fähigkeit der Europäer, das Fremde im Wortsinn an sich heranzulassen.

Indem der Einheimische nun die Aufsplitterung in Gruppen bis auf die atomare Ebene weiterverfolgt - das einzelne Individuum ist dieses Atom - stellt er neben den Europäern und den Inselbewohnern eine dritte Kategorie her: eben die Kategorie des Sich-selbst, Dieses Selbst als Entität weist die Selbstverständlichkeit, dass Raum mit anderen zu teilen ist. als Zumutung von sich.24 Durch seine Linien stellt er die Behauptung auf, das verbürgte Recht auf

23 Diese Verhaltensweise ist darum so interessant, weil sie, àhnlich wie bel den von Todorov beschriebenen Azteken, eine individuelle Reaktion auf die neuen Machtkonstellationen darstellt. Der Einheimische versucht, sich unter den neuen Gegebenheiten zurechtzufinden. Er reagiert auf neue Weise - doch ahnlich wie bei den Azteken wird er sich machtpolitisch nicht durchsetzen könen:

24 Forsters Blick auf die Komik der Situation gewinnt hier eine wesentliche Ambivalenz. Wurde die Konsequenz aus der Absurditat des Kreises gezogen, dann stunde die Dominanz der Europaer selbst in Frage. Indem Forster die Kritik, die der Kreis ubermittelt, vernachlassigt, verschafft er sich eine Position, von der aus er den Kreis als komisch abtun und den Ernst der Kritik in Abrede stellen kann. Durch die Zuschreibung des. Adjektivs skomischi verliert der Einheimische jede Chance darauf, gehört zu werden. 
ein eigenes Territorium, eine Insel auf der Insel, zu haben. Jeder, der den Kreis um seine Person überschretlet, wird auf komischgroteske Weise abgewehrt. Dadurch macht er die Unhaltbarkeit der europäischen Verhaltensweise sichtbar. Unhaltbar sind die Insein auf der Insel, weil weder der Humorist noch die Europaier zu überleben imstande wären, wenn sie die Logik der Abgrenzung konsequent ins Werk setzten. Denn auf einer Insel zu sein, allein, ohne Nebenmenschen, bedeutet eben auch, nicht an dem teilhaben zu können, was dieser andere im Tausch zu bieten hat. Eine Insel, die wie die des Humoristen wenige Schritte Umfang hat, ist dem Tod geweiht, sowohl physisch als auch intellektuell.

Ebenso bringen sich die Europäer, die die Inseln nach den Kategorien hier und dort, 'mein und dein zergliedern, selbst in Gefahr. Die Gefahr besteht darin, dass sie, wenn sie den anderen den Zutritt zu ihrem. Gebiet verweigern, umgekehrt auch den Anspruch auf das verlieren, was jenseits der Grenze liegt. In dem Moment, in dem sie die Bewegungsfreiheit der anderen einschränken, schrănken sie auch ihre eigene ein. Und dies nicht nur, weil die Grenze fur sie selbst bestimmend wird, sondern auch, weil im Falle der Grenzüberschreitung (die man sich selbst problemlos zuerkennen möch(e) mit Gegengewalt zu rechnen ist. Grenzen machen folglich das Leben anstrengend, führen zu Auseinandersetzungen zwischen Hintern und Kanone, wie wir sie oben kennen gelernt hatten - Auseinandersetzungen. in denen stets von Neuem zu beweisen ist, dass mit Recht die Grenzziehung nur von der einen Partei ausgeht, wihrend die andere sich ihr zu beugen hat.

Interessant an dem Einfall des oben beschriebenen Inselbewohners ist, dass seine Aggressivität sich nicht gegen die Europãer rich. tet. sondern gegen seine eigenen Leute. Er scheint ihnen auf diese Weise zu bedeuten, dass er mit den Europäern auf der gleichen Stufe steht. Auf den ersten Blick könnte man meinen, dass er damit den Einheimischen gleicht, die auf den ,Inseln. der Europäer zugelassen worden waren. Doch diese Privilegierten verdankten thre Position der europäischen Politik des divide et impera, unterlagen mithin einer Abhängigkeit von denjenigen, die darüber entschieden, ob sie Zugang haben sollten oder nicht. Der Humorist agiert hingegen vollkommen selbstāndig. Dadurch, dass er seine lnsel nicht von der Europảer Gnaden herstellt, sondern spontan und allein, führt er seinen ehemaligen Nachbarn indirekt die Gefahr vor Augen. die darin besteht, den Zusammenhalt der Inselbewohner durch eine Anpassung an die europäischen Kategorien zu schwächen.

Dass er die anderen abwehrt, ist also nicht ein Zeichen für seine Distanzierung von ihnen, sondern im Gegenteil ein Plâdoyer für grōßere Nähe. Dadurch, dass das, was er abwehrt, bis dahin zur Normalităt gehōrte - den Raum mit anderen teilen -, zeigt er indi- 
rekt, was bereits verloren gegangen ist. Und dieses Verlorene wird umso sichtbarer, je lächerlicher der Umfang des Territoriums ist, das zu verteidigen er sich anschickt. Je kleiner also das, was zum Objekt des Gelächters gemacht werden soll, desto größer die Wirkung auf das Publikum. Denn dieses ist die Instanz, die von der Lächerlichkeit der Darstellung auf die Größenordnung dessen schlieBen soll, was in Wirklichkeit dargestellt werden soll. nămlich die Anmaßung der Europäer, sich selbst als einer höherrangigen Art von Mensch Sonderterritorien zu erobern.

So zeigt sich, dass die Inselbewohner, obwohl sie ein kleines, in sich geschlossenes Territorium bewohnen - eine Insel eben -, in Wirklichkeit weiträumiger denken als die Europäer, die ihre Identitât ûber eine Hypertrophie des Insularen definieren. Zugleich stehen aber auch sie in der Gefahr, die Existenz von Sonderterritorien als Normalität anzuerkennen. denn schließlich ist es ein einziger Inselbewohner, der die Komik winziger Kreise in Szene setzt. In dieser Hinsicht stehen sich die Europäer und die Mehrheit der Inselbewohner dann doch wieder nahe und ist es der Humorist allein, der, eingeschlossen auf seiner Insel, auf die Weitung des Raumes hofft. 